\title{
ON DECOMPOSITION OF SOLUTIONS OF SOME HIGHER ORDER ELLIPTIC EQUATIONS
}

\author{
JUKKA SARANEN
}

We study in $\boldsymbol{R}^{n}$ an equation of the form

$$
P(A) u=f,
$$

where $P(\xi)=\sum_{v=0}^{r} a_{v} \xi^{v}$ denotes a complex polynomial of the degree $r>0$ normalized with $a_{r}=1$ and $A$ is a partial differential operator of the order $2 m$. About the operator

$$
A u=\sum_{0 \leqq|\alpha|,|\beta| \leqq m}(-1)^{|\alpha|} \partial^{\alpha}\left(a_{\alpha \beta} \partial^{\beta} u\right)
$$

we assume that $a_{\alpha \beta}=a_{\alpha \beta}^{0}+r_{\alpha \beta}$, where $a_{\alpha \beta}^{0}$ are constants and the functions $r_{\alpha \beta}$ are infinitely many times differentiable so that they vanish at infinity faster than any negative power of the radius $r=|x|$. It is also required that $a_{\alpha \beta}^{0}=0$ for all multiindices $|\alpha|+|\beta|<2 m$. The polynomial $P(\xi)$ can be written as the product

$$
P(\xi)=\xi^{r_{0}} \prod_{\varrho=1}^{q}\left(\xi-k_{\varrho}^{2}\right)^{r_{\varrho}},
$$

where $0 \neq k_{\underline{Q}}={\nu_{\varrho}}_{\underline{Q}}+i \lambda_{\varrho}, 0 \leqq \arg k_{\varrho}<\pi, k_{\varrho} \neq k_{\tau}, \tau \neq \varrho$. Our aim is to reduce the problem (1) to simpler ones by decomposing any solution of (1) into a certain combination of solutions for equations like

$$
A^{r_{0} w}=g, \quad\left(A-k^{2}\right) w=g, \quad k \neq 0 .
$$

In the cases where these equations have a unique solution in some classes of functions we obtain a unique solution for (1) by fixing the corresponding conditions for the components of $u$. There are some earlier articles which deal with equations of the above polynomial type in unbounded domains. Vekua [8] studied the equation $P(\Delta) u=0$ and also solved an exterior boundary value problem of the Riquier type. Paneyah [6] considered the corresponding inhomogeneous equation in the whole space (for $r_{0}=0$ ). He also pointed out that the Laplace operator could be replaced by a more general second order elliptic operator having constant coefficients. In paper [9] (for $r_{0}=0$ ) Witsch ailowed $A$ to be a uniformly strongly elliptic second order operator whose coefficients approach those of the Laplace operator at infinity. He was also able to give a Fredholm type theorem for the exterior boundary value problem with homogeneous Dirichlet boundary data. 
This article can be considered as a note to the paper of Witsch. With it we would remark that at least the whole space result remains valid if $A$ is a certain higher order operator. Further, the decomposition given in [9] does not cover the case $r_{0}>0$. We make use of the general decomposition for a special case where the dimension of the space $\boldsymbol{R}^{n}$ is large enough; $n \geqq 2 m r_{0}+1$.

The key to the factorization of the solution is a formula which shows how the operator $Q(A)$ with an arbitrary polynomial $Q$ operates on the powers of the differential expression $\Lambda u=x_{i} \partial_{i} u\left(\Lambda^{0} u=u\right)$. For the multi-indices $\alpha=\left(\alpha_{1}, \ldots, \alpha_{n}\right)$ and $e^{i}=\left(e_{1}^{i}, \ldots, e_{n}^{i}\right)$, where $e_{j}^{i}=\delta_{i j}$, we define

Then

$$
\delta\left[\alpha, e^{i}\right]= \begin{cases}1 & \alpha_{i} \geqq 1 \\ 0 & \alpha_{i}=0 .\end{cases}
$$

$$
\partial^{\alpha}\left(x_{i} u\right)=x_{i} \partial^{\alpha} u+\alpha_{i} \delta\left[\alpha, e^{i}\right] \partial^{\alpha-e_{i} u}
$$

holds for all multi-indices $\alpha$. By applying this equality we easily find

$$
\partial^{\alpha}(\Lambda u)=\Lambda \partial^{\alpha} u+|\alpha| \partial^{\alpha} u .
$$

We make use of some notations in [9]. Let $\mathscr{T}$ (resp. $\hat{\mathscr{T}}$ ) denote the class of all functions infinitely many times differentiable which vanish at infinity faster than any negative power of $r$ (resp. which grow more slowly than some positive power of $r$ ). A differential operator whose coefficients are of the class $\mathscr{T}\left(\right.$ resp. $\hat{\mathscr{T}}, C_{0}^{\infty}$ ) is called $\mathscr{T}$-operator (resp. $\hat{\mathscr{T}}-, C_{0}^{\infty}$-operator). We write the operator $A u$ as a sum $A u=$ $A^{0} u+R u$ with

where further

$$
\begin{aligned}
A^{0} u & =\sum_{|\alpha|=|\beta|=m}(-1)^{|\alpha|} a_{\alpha \beta}^{0} \partial^{\alpha+\beta} u, \\
R u & =\sum_{0 \leqq|\alpha|,|\beta| \leqq m}(-1)^{|\alpha|} \partial^{\alpha}\left(r_{\alpha \beta} \partial^{\beta} u\right),
\end{aligned}
$$

$$
R u=\sum_{|\alpha|=|\beta|=m}(-1)^{m} r_{\alpha \beta} \partial^{\alpha+\beta} u+\tilde{M} u
$$

with a $\mathscr{T}$-operator $\tilde{M} u$ of the order $\leqq 2 m-1$. By using (2) we then see that

$$
A(\Lambda u)=\Lambda(A u)+2 m A u+M u
$$

with a $\mathscr{T}$-operator

$$
M=-2 m \tilde{M} u-\sum_{|\alpha|=|\beta|=m}(-1)^{m}\left(\Lambda r_{\alpha \beta}\right) \partial^{\alpha+\beta} u+\tilde{M} \Lambda u-\Lambda \tilde{M} u,
$$

which is at most of the order $\leqq 2 m$. We also see that if the functions $r_{\alpha \beta}$ belong to the class $C_{0}^{\infty}$, then $M$ is a $C_{0}^{\infty}$-operator.

We denote with $d$ the differential operator $d Q(\xi)=\xi Q^{\prime}(\xi) ; d^{\mu}=d\left(d^{\mu-1}\right), d^{0} Q=Q$ in the ring of all polynomials we can generalize formula (3) as follows: 
Lemma 1. Let $Q$ be a complex polynomial of the degree l. For an arbitrary non-negative integer $\mu$ and for a function $u \in C^{\infty}$ we have

$$
Q(A) \Lambda^{\mu} u=\sum_{v=0}^{\mu}\left(\begin{array}{l}
\mu \\
v
\end{array}\right) \Lambda^{v}\left[(2 m d)^{\mu-v} Q\right](A u)+M_{Q(\xi), \mu} u,
$$

where $M_{Q(\xi), \mu}$ is a $\mathscr{T}$-operator of the order $\leqq 2 m l+\mu-1$. If $r_{\alpha \beta} \in C_{0}^{\infty}$, then $M_{Q(\xi), \mu}$ is a $C_{0}^{\infty}$-operator.

Proof. Formula (4) represents only a slight extension of a corresponding result in [9]. For completeness we give the arguments. It is enough to show the validity of (4) for all monomials $Q(\xi)=\xi^{l}$. But formula (3) shows the validity in the case $Q(\xi)=\xi, \mu=1$. The induction on $l$ gives

$$
A^{l}(\Lambda u)=(2 m l+\Lambda) A^{l} u+M_{\xi^{l}, 1} u
$$

because in the induction step we then have

$$
A^{l+1}(\Lambda u)=(2 m(l+1)+\Lambda)\left(A^{l+1} u\right)+A M_{\xi^{l}, 1} u+M_{\xi, 1} A^{l} u,
$$

where $A M_{\xi^{l}, 1}+M_{\xi, 1} A^{l}$ is at most of the order $2 m(l+1)$. Through induction on $\mu$ we obtain

$$
A^{l}\left(\Lambda^{\mu} u\right)=(2 m l+\Lambda)^{\mu} A^{l} u+M_{\xi^{l}, \mu} u,
$$

where in the induction step we have now by (5) and (6)

$$
M_{\xi^{l}, \mu+1}=(2 m l+\Lambda) M_{\xi^{l}, 1}+M_{\xi^{l}, \mu} \Lambda,
$$

which is a $\mathscr{T}$-operator of the order at most $2 m l+\mu$.

By using formula (4) we can prove an extension of a result in [9]:

Theorem 2. If the integer $r_{0}$ is positive, then there exist the $\hat{\mathscr{T}}$-operators $N_{\mu, v}$, $N, M_{\mu, v}^{0}, M^{0}$ and the $\mathscr{T}$-operators $M_{\mu, v}^{e, \sigma}, M^{\varrho, \sigma}$, where $\mu, \varrho=1, \ldots, q ; v=0, \ldots, r_{\mu}-1$; $\sigma=0, \ldots, r_{Q}-1$ and the numbers $B_{j, 0}^{\mu, v}, j=0, \ldots, v$ so that

(i) if $u \in C^{\infty}$ solves equation (1), then the functions

$$
\begin{gathered}
u_{\mu, v}=N_{\mu, v} u, \\
\zeta=N u
\end{gathered}
$$

satisfy the equations

$$
\begin{aligned}
\left(A-k_{\mu}^{2}\right) u_{\mu, v} & =g_{\mu, v}, \\
A^{r_{0} \zeta} & =g
\end{aligned}
$$

with

$$
\begin{aligned}
g_{\mu, v} & =M_{\mu, v}^{0} f+\sum_{Q=1}^{q} \sum_{\sigma=0}^{r_{Q}-1} M_{\mu, v}^{Q, \sigma} u_{Q, \sigma}, \\
g & =M^{0} f+\sum_{Q=1}^{q} \sum_{\sigma=0}^{r_{Q}-1} M^{Q, \sigma} u_{Q, \sigma},
\end{aligned}
$$


and the function $u$ has the representation

$$
u=\zeta+\sum_{\mu=1}^{q} \sum_{v=0}^{r_{\mu}-1} \sum_{j=0}^{v} B_{j, 0}^{\mu, v} \Lambda^{j} u_{\mu, v} .
$$

(ii) If, conversely, the functions $u_{\mu, v}, \zeta \in C^{\infty}$ solve the system of (8) and (9), then the function $u$ defined by (10) satisfies (1), and the functions $u_{\mu, v}, \zeta$ can be calculated from (7).

(iii) The operators $M_{\mu, v}^{\rho, \sigma}$ have the property $M_{\mu, v}^{\varrho, \sigma}=0$ if lexically $(\varrho, \sigma) \geqq(\mu, v)$.

If $r_{\alpha \beta} \in C_{0}^{\infty}$ then the operators $M_{\mu, v}^{o, \sigma}, M^{o, \sigma}$ are $C_{0}^{\infty}$-operators. In the case $r_{0}=0$ the operator $N$ is absent and we must use only (7a), (8a), (9a) and (10) without the function $\zeta$.

Proof. Assume first that $r_{0}=0$. In this case the argument follows [9] without any essential modification. It is also easy to verify from the proof that the operators $M_{\mu, v}^{\varrho, \sigma}$ are $C_{0}^{\infty}$-operators if the functions $r_{\alpha \beta}$ have finite supports. Suppose then that $r_{0}>0$ and let $u \in C^{\infty}$ be a function which satisfies (1). Denote $P(\xi)=\xi^{r_{0}} Q(\xi)$ and $v=A^{r_{0}} u$, which gives $Q(A) v=f$. According to the first case there exist $\hat{\mathscr{T}}$-operators $\hat{N}_{\mu, v}, \hat{M}_{\mu, v}^{0}$ and $\mathscr{T}$-operators $\hat{M}_{\mu, v}^{\varrho, \sigma}$ such that

$$
v=\sum_{\mu=1}^{q} \sum_{v=0}^{r_{\mu}-1} \Lambda^{v} v_{\mu, v}
$$

with

where $\hat{M}_{\mu, v}^{\varrho, \sigma}=0,(\varrho, \sigma) \geqq(\mu, v)$.

$$
\begin{aligned}
& v_{\mu, v}=\hat{N}_{\mu, v} v, \quad\left(A-k_{\mu}^{2}\right) v_{\mu, v}=g_{\mu, v} \\
& g_{\mu, v}=\hat{M}_{\mu, v}^{0} f+\sum_{\varrho=1}^{q} \sum_{\sigma=0}^{r_{\varrho}-1} \hat{M}_{\mu, v}^{\rho, \sigma} v_{\varrho, \sigma},
\end{aligned}
$$

We show now that one can choose the numbers $B_{j, 0}^{\mu, v}, j=0, \ldots, v$ such that the function

$$
w=\sum_{\mu=1}^{q} \sum_{v=0}^{r_{\mu}-1} \sum_{j=0}^{v} B_{j, 0}^{\mu, v} \Lambda^{j} v_{\mu, v}
$$

satisfies the equation

$$
A^{r_{0}} w=v-\sum_{\mu=1}^{q} \sum_{v=0}^{r_{\mu}-1} \hat{M}^{\mu, v} v_{\mu, v}-M^{0} f
$$

where $\hat{M}^{\mu, v}\left(\mu=1, \ldots, q ; v=0,1, \ldots, r_{\mu}-1\right)$ are $\mathscr{T}$-operators and $M^{0}$ is a $\hat{\mathscr{T}}$-operator. Using the formulae (4) and (12) we get

$$
\begin{aligned}
A\left(\Lambda^{j} v_{\mu, v}\right) & =\sum_{\alpha=0}^{j}\left(\begin{array}{c}
j \\
\alpha
\end{array}\right)(2 m)^{j-\alpha} \Lambda^{\alpha}\left(A v_{\mu, v}\right)+M_{\xi, j} v_{\mu, v} \\
& =k_{\mu}^{2} \sum_{\alpha=0}^{j}\left(\begin{array}{l}
j \\
\alpha
\end{array}\right)(2 m)^{j-\alpha} \Lambda^{\alpha} v_{\mu, v}+L_{j}^{\mu, v} g_{\mu, v}+M_{\xi, j} v_{\mu, v}
\end{aligned}
$$

with an $\hat{\mathscr{T}}$-operator $L_{j}^{\mu, v}$. Noting that the product of a $\mathscr{T}$-operator and a $\hat{\mathscr{T}}$-operator is a $\mathscr{T}$-operator we get through the formulae (14), (16) and (13)

$$
A w=\sum_{\mu=1}^{q} \sum_{v=0}^{r_{\mu}-1} \sum_{\alpha=0}^{v} B_{\alpha, 1}^{\mu, v} \Lambda^{\alpha} v_{\mu, v}-M_{1} f-\sum_{\mu=1}^{q} \sum_{v=0}^{r_{\mu}-1} \hat{M}_{1}^{\mu, v} v_{\mu, v}
$$




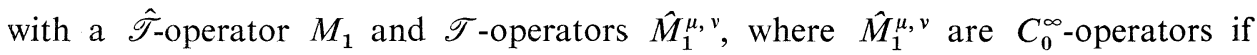
$r_{\alpha \beta} \in C_{0}^{\infty}$. Here the numbers $B_{\alpha, 1}^{\mu, v}$ can be calculated by means of the formula

$$
B_{\alpha, 1}^{\mu, v}=k_{\mu}^{2} \sum_{j=\alpha}^{v} B_{j, 0}^{\mu, v}(2 m)^{j-\alpha}\left(\begin{array}{l}
j \\
\alpha
\end{array}\right), \quad 0 \leqq \alpha \leqq v .
$$

This system has the form

$$
\begin{aligned}
B_{v, 1}^{\mu, v} & =k_{\mu}^{2} B_{v, 0}^{\mu, v}, \\
B_{v-1,1}^{\mu, v} & =k_{\mu}^{2}\left\{B_{v-1,0}^{\mu, v}+2 m\left(\begin{array}{c}
v \\
v-1
\end{array}\right) B_{v, 0}^{\mu, v}\right\}, \\
\vdots & \\
B_{\alpha, 1}^{\mu, v} & =k_{\mu}^{2}\left\{B_{\alpha, 0}^{\mu, v}+2 m\left(\begin{array}{c}
\alpha+1 \\
\alpha
\end{array}\right) B_{\alpha+1,0}^{\mu, v}+\ldots+(2 m)^{v-\alpha}\left(\begin{array}{c}
v \\
\alpha
\end{array}\right) B_{v, 0}^{\mu, v}\right\}
\end{aligned}
$$

and is therefore uniquely solvable; if the numbers $B_{\alpha, 1}^{\mu, v}$ are known, then the numbers $B_{j, 0}^{\mu, v}$ are uniquely defined. When we apply again the operator $A$ to the equation (17) $(l-1)$-times, we can denote generally

$$
A^{l} w=\sum_{\mu=1}^{q} \sum_{v=0}^{r_{\mu}-1} \sum_{\alpha=0}^{v} B_{\alpha, l}^{\mu, v} \Lambda^{\alpha} v_{\mu, v}-M_{l} f-\sum_{\mu=1}^{q} \sum_{v=0}^{r_{\mu}-1} \hat{M}_{l}^{\mu, v} v_{\mu, v}
$$

with a $\hat{\mathscr{T}}$-operator $M_{l}$ and with the $\mathscr{T}$-operators $\hat{M}_{l}^{\mu, v}$ together with the coefficients

$$
B_{\alpha, l}^{\mu, v}=k_{\mu}^{2} \sum_{j=\alpha}^{v} B_{j, l-1}^{\mu, v}(2 m)^{j-\alpha}\left(\begin{array}{l}
j \\
\alpha
\end{array}\right) .
$$

This system is uniquely solvable as above. Choosing at the stage $l=r_{0}$

$$
B_{v, r_{0}}^{\mu, v}=1, \quad B_{\alpha, r_{0}}^{\mu, v}=0, \quad 0 \leqq \alpha<v
$$

and, accordingly, the constants $B_{\alpha, l}^{\mu, v}, l=0, \ldots, r_{0}-1$ such that the equation (20) is valid for every $l=1, \ldots, r_{0}$, we obtain from (14) the function $w$ which satisfies the equation (15) with $M^{0}=M_{r_{0}}, \hat{M}^{\mu, \nu}=\hat{M}_{r_{0}}^{\mu, \nu}$. For the function $\zeta=u-w$ we then have

$$
A^{r_{0}} \zeta=A^{r_{0}} u-A^{r_{0}} w=\sum_{\mu=1}^{q} \sum_{v=0}^{r_{\mu}-1} \hat{M}^{\mu, v} v_{\mu, v}+M^{0} f .
$$

Choosing the operators

$$
\left\{\begin{array}{l}
M^{Q, \sigma}=\hat{M}^{\varrho, \sigma} \\
N_{\mu, v}=\hat{N}_{\mu, v} A^{r_{0}} \\
N=1-\sum_{\mu=1}^{q} \sum_{v=0}^{r_{\mu}-1} \sum_{j=0}^{v} B_{j, 0}^{\mu, v} \Lambda^{j} N_{\mu, \nu} \\
M_{\mu, v}^{0}=\hat{M}_{\mu, v}^{0}, \quad M_{\mu, v}^{e, \sigma}=\hat{M}_{\mu, v}^{\rho, \sigma}
\end{array}\right.
$$


we see that the functions $u_{\mu, \nu}=N_{\mu, \nu} u, \zeta=N u$ satisfy the equations (8) and (9) and that $u$ has the representation (10). If, conversely, $u_{\mu, v}, \zeta \in C^{\infty}$ are functions satisfying the equations (8) and (9), and if $u=\zeta+w$ is defined by (10), we have

$$
A^{r_{0} W}=v-\sum_{\mu=1}^{q} \sum_{v=0}^{r_{\mu}-1} M^{\mu, v} u_{\mu, v}-M^{0} f
$$

with $v=\sum_{\mu=1}^{q} \sum_{v=0}^{r_{\mu}-1} \Lambda^{v} u_{\mu, v}$, and hence further by (9) and (24)

$$
A^{r_{0}} u=v \text {. }
$$

On the other hand, we have from the case $r_{0}=0$ the relations $Q(A) v=f, u_{\mu, \nu}=$ $\hat{N}_{\mu, v} v$ and therefore also

$$
P(A) u=Q(A) v=f
$$

with $u_{\mu, v}=\hat{N}_{\mu, v} v=\hat{N}_{\mu, v} A^{r_{0}} u=N_{\mu, v} u$. Finally we obtain

$$
\zeta=u-\sum_{\mu=1}^{q} \sum_{v=0}^{r_{\mu}-1} \sum_{j=0}^{v} B_{j, 0}^{\mu, v} \Lambda^{j} u_{\mu, v}=N u .
$$

Let us assume that the coefficients satisfy $a_{\alpha \beta}(x)=\bar{a}_{\beta \alpha}(x)$ and that the operator $A$ is uniformly strongly elliptic so that

$$
\sum_{|\alpha|,|\beta|=m} a_{\alpha \beta}(x) \xi^{\alpha} \xi^{\beta} \geqq c|\xi|^{2 m}, \quad c>0
$$

for every $x \in \boldsymbol{R}^{n}, \xi \in \boldsymbol{R}^{n}$. For a given $A$ we define for every complex number $z$ an operator $A_{0}+z$ in $L^{2}=L^{2}\left(\boldsymbol{R}^{n}\right)$ with the domain

$$
D\left(A_{0}+z\right)=\left\{u \in H^{m} \mid \exists f \in L^{2}: \forall \varphi \in C_{0}^{\infty} \quad B_{z}(u, \varphi)=(f \mid \varphi)_{0}\right\}
$$

(which in fact is independent of $z$ ) and define further $A_{0} u+z u=f$ for $u \in D\left(A_{0}+z\right)$. Here we have used the defining formula

$$
B_{z}(u, \varphi)=\sum_{0 \leqq|\alpha,|,|\beta| \leqq m}\left(a_{\alpha \beta} \partial^{\beta} u \mid \partial^{\alpha} \varphi\right)_{0}+z(u \mid \varphi)_{0}
$$

for the sesquilinear form $B_{z}: H^{m} \times H^{m} \rightarrow \boldsymbol{C}$ with $H^{m}=H^{m}\left(\boldsymbol{R}^{n}\right)=H_{0}^{m}\left(\boldsymbol{R}^{n}\right)$. The operator $A_{0}$ is symmetric and by the inequality of Gårding ([1])

$$
\operatorname{Re} B_{-k^{2}}(u, u) \geqq c_{1}\|u\|_{m}^{2}-c_{2}\|u\|_{0}^{2}
$$

with $c_{1}>0, c_{2} \geqq 0$ as well as by the formula

$$
\operatorname{Im} B_{-k^{\mathbf{3}}}(u, u)=-2 \lambda x\|u\|_{0}^{2}
$$

we get in the case $\lambda \neq 0, x \neq 0, k=x+i \lambda$ for a sufficiently small number $0<\eta \leq 1$ for all $u \in H^{m}$

$$
\begin{aligned}
\left|B_{-k^{\natural}}(u, u)\right| & \geqq \frac{1}{\sqrt{2}}\left(\eta\left|\operatorname{Re} B_{-k^{2}}(u, u)\right|+\left|\operatorname{Im} B_{-k^{\mathfrak{s}}}(u, u)\right|\right) \\
& \geqq c_{3}\|u\|_{m}^{2}
\end{aligned}
$$


with a positive number $c_{3}=c_{3}(\eta)$. According to well-known arguments it then holds for the ranges $R\left(A_{0}-k^{2}\right)=L^{2}$ if $\varkappa \neq 0, \lambda \neq 0$. The operator $A_{0}$ is therefore selfadjoint ([2]). We assume that the operator $A_{0}$ is also positive, in other words, $\left(A_{0} u \mid u\right)_{0} \geqq 0$ for every $u \in D\left(A_{0}\right)$. If we then take $f \in L^{2}$ and $k=i \lambda, \lambda>0$, there exists exactly one function $u \in D\left(A_{0}\right)$ with $A_{0} u-k^{2} u=f$. If in addition $f \in C^{\infty}$, then the function $u$ is also regular and satisfies the equation $A u-k^{2} u=f$ in the classical sense.

In the case $k>0$ we utilize a result of Vainberg ([7]). Let

$$
Q_{k}(\xi)=\sum_{|\alpha|=|\beta|=m} a_{\alpha \beta}^{0} \xi^{\alpha+\beta}-k^{2}
$$

be the characteristic polynomial of the operator $A^{0}-k^{2}$ and suppose that $A^{0}$ is elliptic with $a_{\alpha \beta}^{0}=\overline{a_{\alpha \beta}^{0}}$. Denote by $N_{k}$ the set of the zeros for $Q_{k}$ in $\boldsymbol{R}^{n}$. It is then easy to see that $N_{k}$ is compact, connected and non-empty, so that $\operatorname{grad}_{\xi} Q(\xi) \neq 0$ if $\xi \in N_{k}$. Hence $N_{k}$ is also a smooth $(n-1)$-dimensional surface. In order to use [7] we must consider operators where the part $R u$ containing variable coefficients depends on a parameter. Let therefore $R^{0}$ be a differential operator of the order at most $2 m$ such that its coefficients are in $C_{0}^{\infty}$. Denote with $D$ the open set of points $\varepsilon \in C$ where the operator $A^{0}+\varepsilon R^{0}$ is uniformly strongly elliptic and let $D_{0}$ stand for the connected component of $D$ which contains the origin. If the total curvature (Gaussian curvature) vanishes at no point of $N_{k}$, then for any $f \in C_{0}^{\infty}$ the equation

$$
\left(A^{0}+\varepsilon R^{0}\right) u-k^{2} u=f
$$

has for almost all values $\varepsilon \in D_{0}$ (apart from a discrete set), especially including $\varepsilon=0$, a unique solution $u \in C^{\infty}$ satisfying for $r>0$

$$
|u(x)| \leqq C r^{(1-n) / 2}, \quad\left|\frac{\partial}{\partial r} u-i \mu(\omega) u(x)\right| \leqq C r^{-n / 2}
$$

with a $C>0$. Here $\mu(\omega)=(\sigma(\omega) \mid \omega)$ with $\omega=x / r$ and $\sigma(\omega)$ is the point on the surface where a continuously chosen normal has the same direction as $\omega$.

To put all the foregoing things together we require the following:

$$
\text { 1) } A^{0} u=(-1)^{m} \sum_{|\alpha|=|\beta|=m} a_{\alpha \beta}^{0} \partial^{\alpha+\beta} u, \quad a_{\alpha \beta}^{0}=\overline{a_{\beta \alpha}^{0}} \text {, }
$$

is strongly elliptic.

2) The total curvature of the surfaces $N_{k}, k>0$ does not vanish.

3) $R^{0} u=\sum_{|\alpha|,|\beta| \leqq m}(-1)^{|\alpha|} \partial^{\alpha}\left(r_{\alpha \beta}^{0} \partial^{\beta} u\right), \quad r_{\alpha \beta}^{0}=\overline{r_{\beta \alpha}^{0}} \in C_{0}^{\infty}$

satisfies $\left(R^{0} \varphi \mid \varphi\right)_{0} \geqq 0$ for every $\varphi \in C_{0}^{\infty}$.

In particular, assumptions 1) and 3) imply that the corresponding operator $\left(A^{0}+\varepsilon R^{0}\right)_{0}$ in $L^{2}$ is positive for every $\varepsilon \geqq 0$. 
Theorem 3. Let $A=A^{0}+\varepsilon R^{0}$ be a differential operator such that assumptions 1)-3) are valid. Then apart from a discrete set of points $\varepsilon \geqq 0$ including $\varepsilon=0$ the equation $P(A) u=f \in C_{0}^{\infty}$ has for any polynomial $P(\xi)$ which does not vanish at the origin, a unique solution $u \in \hat{\mathscr{T}}$ such that $N_{\mu v} u \in H^{m}$ for $\operatorname{Im} k_{\mu}>0$ and $N_{\mu v} u$ satisfies (26) for $k_{\mu}>0$.

Proof. It suffices to note that the solution $u$ for the equation $\left(A-k^{2}\right) u=g \in C_{0}^{\infty}$, where $u \in H^{m}$ if $\operatorname{Im} k>0$ and where $u$ satisfies (26) if $k>0$, belongs also to the class $\hat{\mathscr{T}}$. In the first case the characteristic polynomial for $A^{0}-k^{2}$ does not have real zeros and there exists a fundamental solution $E$ for the equation $A^{0} u-$ $k^{2} u=h$ which approaches zero exponentially at infinity ([5]). In the second case the equation $A^{0} u-k^{2} u=h$ has a fundamental solution $E$ which satisfies (26) for $|x| \geqq R>0$ so that every solution $u$ which satisfies also (26) has the form $u=E * h$ ([7]). Convolving the equation $A^{0} u(x)+\varepsilon R^{0} u(x)=g(x)$ with $E$ we obtain

$$
u(x)=-\varepsilon \int_{|y| \leq R_{0}} E(x-y) R^{0} u(y) d y+\int_{|y| \leqq R_{0}} E(x-y) g(y) d y
$$

if the functions $r_{\alpha \beta}, g$ vanish for $|x| \geqq R_{0}$. For $|x| \geqq 2 R_{0}$ we get

and therefore

$$
\partial^{\alpha} u(x)=-\varepsilon \int_{|y| \leqq R_{0}} E(x-y) \partial^{\alpha} R^{0} u(y) d y+\int_{|y| \leqq R_{0}} E(x-y) \partial^{\alpha} g(y) d y
$$

$$
\left|\partial^{\alpha} u(x)\right| \leqq c|x|^{-(n-1) / 2},
$$

which implies $u \in \hat{\mathscr{T}}$. In the case $\operatorname{Im} k>0$ we conclude even $u \in \mathscr{T}$. We can now obtain the unique solution $u \in \hat{\mathscr{T}}$ by solving the system of (8a) and (9a), starting from the indices $(\mu, v)=(1,0)$ and moving in the general step from the pair $(\mu, v)$ to the pair $(\mu, v+1)$ if $v \leqq r_{\mu}-2$ and to $(\mu+1,0)$ if $v=r_{\mu}-1$. Because of (iii) the function $g_{\mu \nu}$ can always be calculated from the known functions and $g_{\mu \nu}$ belongs to the class $C_{0}^{\infty}$ since $f \in C_{0}^{\infty}$ and $M_{\mu, v}^{e, \sigma}$ are $C_{0}^{\infty}$-operators.

We are not able to solve an equation of the type $A^{r_{0}} u=f$ uniquely for a general $r_{0}>0$. In the following we assume that the dimension of the space $\boldsymbol{R}^{n}$ is suffciently large; $n \geqq 2 m r_{0}+1$. Let $H_{k}$ denote the completion of $C_{0}^{\infty}$ with respect to the norm $\|\cdot \mid\|_{k}$,

$$
\|u \mid\|_{t}^{2}=\sum_{i=0}^{k} \sum_{|\alpha|=v}\left\|\frac{\partial^{\alpha} u}{(1+|x|)^{k-v}}\right\|_{0}^{2},
$$

and let $|\cdot|_{k, G}$ be the usual seminorm,

With $|u|_{k}=|u|_{k, R^{n}}$ we get

$$
|u|_{k, G}^{2}=\sum_{|\alpha|=k}\left\|\partial^{\alpha} u\right\|_{0, G}^{2} .
$$

Lemma 4. Let $n \geqq 2 k+1, k>0$. Then there exists a constant $\gamma=\gamma(n, k)>0$ such that the inequality

$$
|| u||_{k} \leqq \gamma|u|_{k}
$$

is valid for every $u \in H_{k}$. 
Proof. For the technique of the following argumentation see [4]. Denote $B(r, \varrho)=$ $\left\{x \in \boldsymbol{R}^{n}|r<| x \mid<\varrho\right\}$ with $0 \leqq r<\varrho \leqq \infty$. Take $1 \leqq l \leqq k, B=B(1 / 2, \infty)$ and $v \in C_{0}^{\infty}(B)$. Partial integration gives

$$
\left\|\frac{\nabla v}{|x|^{l-1}}+s \frac{x}{|x|} \frac{v}{|x|^{l}}\right\|_{0, B}^{2}=\left\|\frac{\nabla v}{|x|^{l-1}}\right\|_{0, B}^{2}+s[s-(n-2 l)]\left\|\frac{v}{|x|^{l}}\right\|_{0, B}^{2}
$$

for every real number $s$. Choosing $s=n-2 l \neq 0$ we get

$$
s\left\|\frac{v}{|x|^{l}}\right\|_{0, B} \leqq\left\|\frac{\nabla v}{|x|^{l-1}}+s \frac{x}{|x|} \frac{v}{|x|^{l}}\right\|_{0, B}+\left\|\frac{\nabla v}{|x|^{l-1}}\right\|_{0, B} \leqq 2\left\|\frac{\nabla v}{|x|^{l-1}}\right\|_{0, B}
$$

and further by induction

$$
\left\|\frac{v}{|x|^{k}}\right\|_{0, B} \leqq c_{1}|v|_{k, B} .
$$

We fix a test function $\varphi$ supported in $B_{1}(0,2)=\left\{x \in \boldsymbol{R}^{n}|| x \mid<2\right\}$ such that $\varphi(x) \equiv 1$, $|x| \leqq 1$. By using (29) and ([4]: Lemma 3.6) we obtain

$$
\begin{aligned}
\left\|\frac{(1-\varphi) u}{(1+|x|)^{k}}\right\|_{0} & \leqq c_{2}\left\|\frac{(1-\varphi) u}{|x|^{k}}\right\|_{0, B(1 / 2, \infty)} \leqq c_{3}|(1-\varphi) u|_{k, B(1 / 2, \infty)} \\
& \leqq c_{4}\left(\|u\|_{k-1, B(1,2)}+|u|_{k}\right) \leqq c_{5}|u|_{k}
\end{aligned}
$$

for $n \geqq 2 k+1$. On the other hand, the Poincaré inequality gives

$$
\left\|\frac{\varphi u}{(1+|x|)^{k}}\right\|_{0} \leqq c_{6}|\varphi u|_{k, B_{1}(0,2)} \leqq c_{7}\left(\|u\|_{k-1, B(1,2)}+|u|_{k, B_{1}(0,2)}\right) \leqq c_{8}|u|_{k} .
$$

From (30), (31) we get

$$
\left\|\frac{u}{(1+|x|)^{k}}\right\|_{0} \leqq c_{9}|u|_{k}
$$

which easily implies (28) in $C_{0}^{\infty}$ and thus in $H_{k}$.

To solve the equation $A^{r_{0}} u=f$ we define a $\|\cdot \cdot\| \|_{m r_{0}}$-bounded sesquilinear form $B_{r_{0}}: H_{m r_{0}} \times H_{m r_{0}} \rightarrow C$ by the formulae

$$
\begin{aligned}
B_{1}(u, v) & =\sum_{0 \equiv|\alpha|,|\beta| \leqq m}\left(a_{\alpha \beta} \partial^{\beta} u \mid \partial^{\alpha} v\right)_{0}, \\
B_{2 l}(u, v) & =\left(A^{l} u \mid A^{l} v\right)_{0}, \quad l=1,2, \ldots, \\
B_{2 l+1}(u, v) & =B_{1}\left(A^{l} u, A^{l} v\right), \quad l=1,2, \ldots .
\end{aligned}
$$

When we write $A=A^{0}+\varepsilon R^{0}$ and $r_{0}=2 l$ the form $B_{r_{0}}(u, v)$ becomes

$$
B_{r_{0}}(u, v)=\left(\left(A^{0}\right)^{l} u \mid\left(A^{0}\right)^{l} v\right)_{0}+\varepsilon \widetilde{B}_{r_{0}}(u, v ; \varepsilon),
$$

where $\widetilde{B}_{r_{0}}(u, v ; \varepsilon)$ is a $\||\cdot|\|_{m r_{0}}$-bounded sesquilinear expression with

$$
\begin{aligned}
\sup _{0 \leqq \varepsilon \leqq 1}\left|\widetilde{B}_{r_{0}}(u, v ; \varepsilon)\right| & \leqq c_{10}|||u|\left\|_{m r_{0}}|||v|\right\|_{m r_{0}} \\
& \leqq c_{11}|u|_{m r_{0}}|v|_{m r_{0}}
\end{aligned}
$$


for every $u, v \in C_{0}^{\infty}$. On the other hand, we have in $C_{0}^{\infty}$

$$
\left(\left(A^{0}\right)^{l} u \mid\left(A^{0}\right)^{l} u\right)_{0} \geqq c_{12}|u|_{m r_{0}}^{2}
$$

([1]: Lemma 7.7). Hence for a sufficiently small $\varepsilon_{0}>0$

$$
B_{r_{0}}(u, u) \geqq\left(c_{12}-\varepsilon c_{10}\right)|u|_{m r_{0}}^{2} \geqq c_{13} \mid\|u\|_{m r_{0}}^{2}
$$

holds with a positive number $c_{13}$ for every $0 \leqq \varepsilon \leqq \varepsilon_{0}, u \in H_{m r_{0}}$. We can prove the inequality (32) analogously in the case $r_{0}=2 l+1, l \geqq 0$. If $f \in \mathscr{T}$, then the scalar product $(u \mid f)_{0}$ is continuous in $H_{m r_{0}}$ :

$$
\begin{aligned}
\left|(u \mid f)_{0}\right| & \leqq\left\|(1+|x|)^{-m r_{0}} u\right\|_{0}\left\|(1+|x|)^{m r_{0}} f\right\|_{0} \\
& \leqq\|u\|\left\|_{m r_{0}}\right\|(1+|x|)^{m r_{0}} f \|_{0} ;
\end{aligned}
$$

for this reason the equation $A^{r_{0}} u=f$ has a unique solution $u \in H_{m r_{0}} \cap C^{\infty}$ by the theorem of Lax-Milgram.

Theorem 5. Let $A=A^{0}+\varepsilon R^{0}$ be a differential operator such that assumptions 1)-3) are valid and let $n \geqq 2 m r_{0}+1$. Then apart from a discrete set of points $0 \leqq \varepsilon \leqq \varepsilon_{0}$ including $\varepsilon=0$ the equation $P(A) u=f \in C_{0}^{\infty}$ for a sufficiently small $\varepsilon_{0}>0$ has a unique solution $u \in \hat{\mathscr{T}}$; for $\operatorname{Im} k_{\mu}>0 \quad N_{\mu v} \in H^{m}$ and for $k_{\mu}>0 \quad N_{\mu \nu} u$ satisfies (26) and $\mathrm{Nu}$ belongs to $\mathrm{H}_{m r_{0}}$.

Proof. We only have to show that a solution $u \in H_{m r_{0}} \cap C^{\infty}$ of $A^{r_{0}} u=f \in C_{0}^{\infty}$ belongs to $\hat{\mathscr{T}}$. But this follows exactly as in [9] because we have

$$
\|u\|_{m r_{0}} \leqq c\left\|(1+|x|)^{m r_{0}} f\right\|_{0}
$$

with a number $c$ independent of $u$.

It may be pointed out that if [3] is used instead of [7], a stronger result can be obtained in the second order case.

\section{References}

[1] Agmon, S.: Lectures on elliptic boundary value problems. - D. Van Nostrand Company, Inc., New York-London-Toronto, 1965.

[2] Hellwig, G.: Differential operators in mathematical physics. - Addison-Wesley, Reading, Massachusetts-Palo Alto-London-Don Mills, Ontario, 1967.

[3] JÄGER, W.: Zur Theorie der Schwingungsgleichung mit variablen Koeffizienten in Außengebieten. - Math. Z. 102, 1967, 62-68.

[4] NeittaAnmäKI, P.: Randwertaufgaben zur Plattengleichung. - Ann. Acad. Sci. Fenn. Ser. A I Math. Dissertationes 16, 1978, 1-71.

[5] Palamodov, V. P.: On conditions at infinity for correct solvability of a certain class of equations of the form $P\left(i \frac{\partial}{\partial x} u\right)=f$. - Dokl. Akad. Nauk. SSSR 129, 1959, 740-743 (Russian).

[6] Paneyah, B. P.: Existence and uniqueness of the solution of the $n$-metaharmonic equation on an unbounded space. - Vestnik Moscov. Univ. Ser. Mat. Meh. Astronom. Fiz. Him. 5, 1959, 123-135 (Russian). 
[7] Vainberg, B. R.: Principles of radiation, limit absorption and limit amplitude in the general theory of partial differential equations. - Russian Math. Surveys 21:3, 1966, 115193.

[8] VeKUA, I. N.: New methods in solving elliptic boundary value problems. - Appendix II, Amsterdam, 1967.

[9] Witsch, K. J.: Radiation conditions and the exterior Dirichlet problem for a class of higher order elliptic operators. - J. Math. Anal. Appl. 54, 1976, 820-839.

\author{
University of Jyväskylä \\ Department of Mathematics \\ Sammonkatu 6 \\ SF-40100 Jyväskylä 10 \\ Finland
}

Received 17 November 1978 